\title{
Do Basic Psychomotor Skills Transfer Between Different Image-based Procedures?
}

\author{
Sonja N. Buzink • Richard H. M. Goossens • \\ Erik J. Schoon · Huib de Ridder · Jack J. Jakimowicz
}

Published online: 12 February 2010

(c) The Author(s) 2010. This article is published with open access at Springerlink.com

\begin{abstract}
Background Surgical techniques that draw from multiple types of image-based procedures (IBP) are increasing, such as Natural Orifice Transluminal Endoscopic Surgery, fusing laparoscopy and flexible endoscopy. However, little is known about the relation between psychomotor skills for performing different types of IBP. For example, do basic psychomotor colonoscopy and laparoscopy skills interact? Methods Following a cross-over study design, 29 naïve endoscopists were trained on the Simbionix GI Mentor and the SimSurgery SEP simulators. Group C $(n=15)$ commenced with a laparoscopy session, followed by four colonoscopy sessions and a second laparoscopy session. Group L $(n=14)$ started with a colonoscopy session, followed by four laparoscopy sessions and a second colonoscopy session.

Results No significant differences were found between the performances of group $\mathrm{L}$ and group $\mathrm{C}$ in their first training sessions on either technique. With additional
\end{abstract}

Part of the work described in this article was presented at the annual congress of the Society of American Gastrointestinal Endoscopic Surgeons 2008 in Philadelphia, Pennsylvania.

S. N. Buzink $(\bowtie)$ · R. H. M. Goossens · H. de Ridder ·

J. J. Jakimowicz

Faculty of Industrial Design Engineering, Delft University of

Technology, Landbergstraat 15, 2628 CE Delft, The Netherlands

e-mail: s.n.buzink@tudelft.nl

S. N. Buzink · J. J. Jakimowicz

Department of Surgery, Catharina Hospital Eindhoven, Michelangelolaan 2, 5623 EJ Eindhoven, The Netherlands

\section{E. J. Schoon}

Department of Gastroenterology, Catharina Hospital Eindhoven, Michelangelolaan 2, 5623 EJ Eindhoven, The Netherlands colonoscopy training, group $\mathrm{C}$ outperformed group $\mathrm{L}$ in the second laparoscopy training session on the camera navigation task.

Conclusions Overall, training in the basic colonoscopy tasks does not affect performance of basic laparoscopy tasks (and vice versa). However, to limited extent, training of basic psychomotor skills for colonoscopy do appear to contribute to the performance of angled laparoscope navigation tasks. Thus, training and assessment of IBP typespecific skills should focus on each type of tasks independently. Future research should further investigate the influence of psychometric abilities on the performance of IBP and the transfer of skills for physicians who are experienced in one IBP type and would like to become proficient in another type of IBP.

\section{Introduction}

In recent years, the performance of medical procedures has become increasingly more technology driven and technology dependent, with a substantial number of procedures now performed image-based [1, 2]. Image-based procedures (IBP) involve all types of medical procedures that allow therapeutic intervention while the operating field is primarily perceived via an intraoperatively obtained image on a display, such as in laparoscopy [3]. In comparison to traditional open procedures, the conduct of IBP necessitates additional skills. Extensive training is needed to achieve the required proficiency level, a great deal of which involves training of operator interaction with the IBP interface [4-7]. In addition to the visual information presented on the display and associated IBP-dedicated equipment, the interface also includes control of the tools to perform the procedure. In most IBP, hand-eye 
coordination of the instruments is counterintuitive and involves considerable visuomotor translations; in addition, the operator often has to deal with a two-dimensional representation on the display of the three-dimensional operating field $[1,4,6]$. IBP are relatively novel procedures, and much still needs to be learned about the human factors of the interaction, additionally required skills, training, and proficiency assessment for the already wellestablished IBP types, such as laparoscopy and flexible endoscopic intraluminal interventions [7-10]. Simultaneously, because of technological innovations, the field of IBP also continues to develop, with increasingly complex procedures being performed image-based procedures. Most studies to date have focused on the performance of a particular technique or task for the well-established IBP types. On a higher level of skills acquisition, little is known about IBP skills in general and the relation between type-specific skills for different IBP.

Proper insight into the fundamental aspects of performing IBP is indispensable to develop training programs, proficiency assessment tools, and trainee selection criteria. Nowadays, flexible endoscopic intraluminal interventions, such as colonoscopy, as well as laparoscopic operations, are widespread procedures. Training in basic skills for both types of procedures can well be done preclinically on virtual reality (VR) simulators [9-13]. Colonoscopy and laparoscopy are two IBP that have elements in common, such as the use of video as the imaging technique. On other elements however, they differ considerably, such as the hand-eye coordination and visuomotor translation to manipulate the camera and surgical instruments.

Gastrointestinal surgeons are often accustomed to performing both laparoscopy and colonoscopy, and the existing assumption is that experience in one of those techniques is of considerable benefit when learning the other [14-16]. However, the relation between laparoscopy and flexible intraluminal endoscopy skills has hardly been studied. So far, the only comparative study on these skills is the one by Adamsen et al. [17], who found a positive correlation between basic skills observed in simulated laparoscopy and basic skills observed in simulated flexible gastrointestinal endoscopy. With the advent of operative techniques that draw from both laparoscopy and flexible intraluminal endoscopy, such as Natural Orifice Transluminal Endoscopic Surgery (NOTES), the lack of knowledge on the relation between skills on these techniques becomes even more apparent [14, 18, 19].

The aim of the present study was to explore the influence of training in basic psychomotor skills for colonoscopy on the performance of basic laparoscopy tasks, and the influence of training in basic psychomotor skills for laparoscopy on the performance of basic colonoscopy tasks. For this purpose two groups of medical trainees were trained in either basic colonoscopy or basic laparoscopy tasks following a cross-over study design. After the first and fourth training sessions for each technique, the influence of the colonoscopy training on their laparoscopy performance and the influence of the laparoscopy training on their colonoscopy performance were assessed (Fig. 1). In addition, the progression in performance within each group over the course of the training program on both image-based techniques was analyzed. Thus, the research was based on several questions: Does the performance of basic IBP tasks improve within two (RQ1a) and four (RQ1b) training sessions by training on a dedicated simulator for that image-based technique (Fig. 2 and Table 1)? And, does the performance of basic IBP tasks improve within two training sessions (RQ2) by training on a dedi-

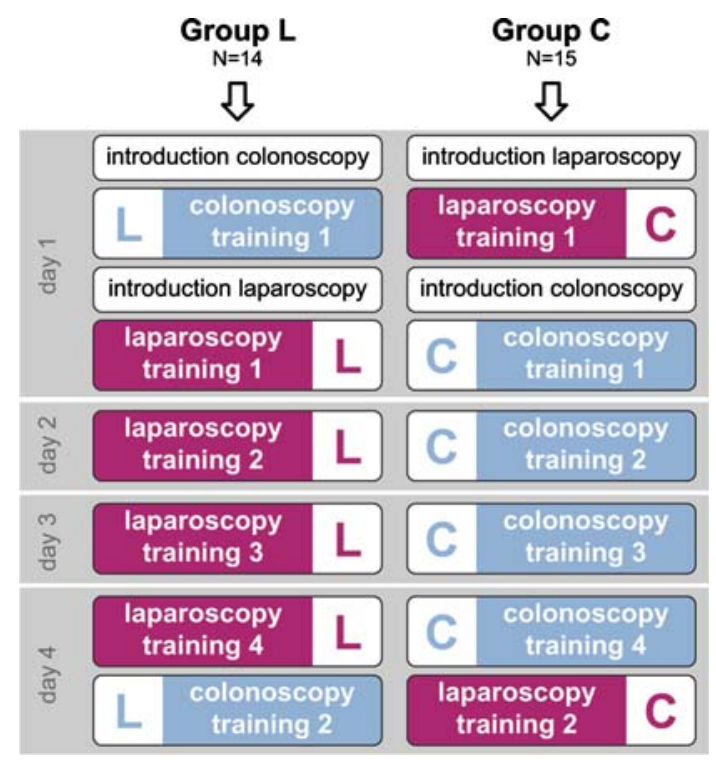

Fig. 1 The study protocol

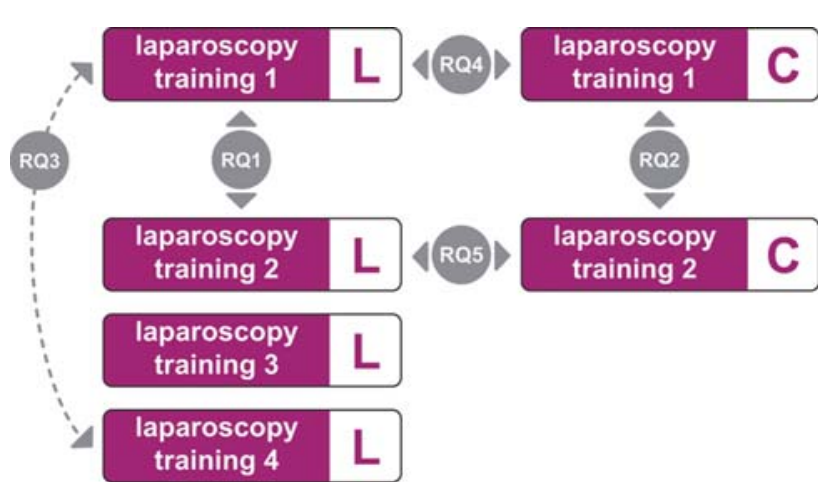

Fig. 2 Overview of the research questions (RQ) comparing performance on the laparoscopy tasks. (For the research questions comparing the performance on the colonoscopy tasks, replace laparoscopy by colonoscopy) 
Table 1 Overview of the research questions and performance comparisons from the perspective of the laparoscopy tasks

\begin{tabular}{lll}
\hline Research question & Compared performance scores \\
\hline RQ 1a. & $\begin{array}{c}\text { Does the performance of basic laparoscopy tasks improve over } \\
\text { the course of two laparoscopy training sessions? }\end{array}$ & Within group L: Laparoscopy training sessions 1-2 \\
RQ 1b. & $\begin{array}{l}\text { Does the performance of basic laparoscopy tasks improve over } \\
\text { the course of four laparoscopy training sessions? }\end{array}$ & Within group L: Laparoscopy training sessions \\
RQ 2. & $\begin{array}{l}\text { Does the performance of basic laparoscopy tasks improve over } \\
\text { the course of two laparoscopy training sessions? }\end{array}$ & Within group C: Laparoscopy training sessions 1-2 \\
RQ 3. & $\begin{array}{l}\text { Does one colonoscopy training session influence } \\
\text { the performance on basic laparoscopy tasks? }\end{array}$ & Laparoscopy training session 1 group C versus \\
RQ 4. & $\begin{array}{l}\text { Do three additional colonoscopy training sessions influence } \\
\text { the performance in a second laparoscopy training session? }\end{array}$ & $\begin{array}{l}\text { Laparoscopy training session } 1 \text { group L } \\
\text { Laparoscopy training session } 2 \text { group C versus }\end{array}$ \\
\hline
\end{tabular}

${ }^{a}$ For the colonoscopy tasks, replace laparoscopy by colonoscopy and colonoscopy by laparoscopy

cated simulator for that image-based technique, while inbetween performing four training session in another IBP technique? Next, by comparing the performances of the two groups with a different training history (one group with and the other group without additional training in the other image-based technique), the transfer of skills between the two image-based techniques was investigated. Does training in basic laparoscopy skills affect the performance on basic colonoscopy tasks? And, vice versa? For example, does the interaction between these skills result in a significant difference in laparoscopy performance after one colonoscopy training session (RQ3)? And what is the difference in colonoscopy performance after three additional laparoscopy training sessions (RQ4)?

\section{Methods and materials}

To enhance the clarity of the article we present the methods from the perspective of the influence of colonoscopy training on the performance of basic laparoscopy task. However, as described above, we also investigated the transfer of skills in the opposite direction. By adding the phrase "and vice versa" we refer to the transfer of skills from laparoscopy training to colonoscopy performance.

In this study, 29 medical trainees with no clinical experience in colonoscopy and laparoscopy took part (Fig. 1). The participants received information about the nature of the study and the activities involved and they filled out an informed consent form. The participants were stratified by their overall performance on psychometric ability tests and then randomly allotted to one of two groups for the simulator training: group $\mathrm{L}(n=14)$ or group C $(n=15)$. During the simulator training sessions the participants trained in manipulation of the flexible endoscope and navigation to the cecum on the Simbionix GI Mentor II VR simulator (software version 2.7.4.0,
Simbionix Corporation, Cleveland, OH) (colonoscopy training) and in bimanual tissue manipulation and 30degree angled laparoscope navigation on the SimSurgery SEP VR simulator (SimSurgery AS, Norway) (laparoscopy training).

\section{Colonoscopy training}

The GI Mentor II VR simulator provides different modules for training in basic flexible endoscopy skills and lower and upper endoscopy procedures. In the present study, the EndoBubble Level 1 (EB L1) task and case 3 of VR Colonoscopy Module I (VRC I-3) were performed in each session. To avoid bias, each of the training sessions also involved performance on multiple different VR colonoscopy cases, and the participants were not notified about the repetitive nature of VRC I-3. The number and order of the colonoscopy training tasks were adopted from the training program used in the study by Buzink et al. [11]. The assignment given to the participants was to perform the EB L1 task as accurately and quickly as possible. The assignment for the VRC cases was to visualize the cecum as quickly as possible, with as little patient discomfort as possible. When the participant reached the cecum, the VRC task was considered accomplished. Participants were instructed not to identify or treat the pathologies presented in the cases. All participants performed the colonoscopy tasks single-handedly, without nurse-assistance for scope insertion.

After each task, the simulator presents the scores and statistics on the performance. In the present study the scores used to analyze performance on the VRC I-3 tasks were these: time to accomplish the task, the percentage of time the virtual patient was in excessive pain, and the percentage of time spent with clear view. For the EB L1 task: time to accomplish the task, number of balloons popped, and number of wall collisions were used. 
Laparoscopy training

For the laparoscopy tasks, the SEP simulation software (SimSurgery AS, Norway) was used. It includes a range of tasks in a VR environment to train different laparoscopy skills. The tasks used in the present study were the Camera Navigation (CN) task with a 30-degree angled laparoscope and the Place Arrow (PA) task, which represents a bimanual tissue manipulation task. The structure of the laparoscopy training was based on the training program used in the study by Buzink et al. [12]. The assignment given to the participants was to perform each task as accurately and quickly as possible. The software provides the scores and a graphical representation of the performance after each task. To analyze the performance of the trainees, their scores on time to accomplish task and the total tip trajectory of the instruments were used, together with the number of lost (over stretched) arrows (PA task) and the number of times the target was lost out of view $(\mathrm{CN}$ task). To analyze and compare the performances during the laparoscopy training, the last repetition in every session of the $\mathrm{CN}$ task and PA task were used as representative. The SEP software was used in combination with a Xitact/ Mentice IHP hardware platform (Mentice AB/Xitact SA, Morges, Switzerland), because the look and feel of the camera tool was considered to resemble the handling of a laparoscope more closely. Although the Xitact/Mentice IHP hardware platform can provide force feedback, the tasks used in the study do not require such feedback. The settings of the instrument trocars were therefore adjusted to compensate for the additional effort required to insert the instruments in the trocars.

\section{Protocol}

The training consisted of six simulator training sessions within one week (Fig. 1). Prior to the first training session, the participants filled out a questionnaire about demographics and their general medical and endoscopy experience. The participants received a standardized introduction to familiarize them with the techniques, simulators, and tasks in preparation for the training sessions. During the introduction it was clearly stated that the researchers were not affiliated with the manufacturers of the simulators and that all data would be analyzed anonymously.

On the first test day group $\mathrm{C}$ started with one laparoscopy training session to assess baseline laparoscopy performance, followed by one colonoscopy training session. Within the following six days, they performed three more colonoscopy training sessions and afterwards a second laparoscopy training session. On the first day, group L started with a colonoscopy training session and subsequently one laparoscopy training session. Within the subsequent six days this was followed by three more laparoscopy training sessions and finally a second colonoscopy training session. No feedback on performance was given other than that produced by the simulators during or after the tasks. Questions related to the use of the tools were answered, whenever asked during the training program, but no instructions were given on how to optimize performance.

Data analysis

We compared the performances in the training sessions within and between the groups with SPSS 16.0 software (SPSS Inc., Chicago, IL) (Fig. 2, Table 1). On the basis of one-way repeated measures multivariate analysis of variance (MANOVA) and analysis of variance (ANOVA) tests, we assessed the performance improvement on each task within group $\mathrm{L}$ and within group $\mathrm{C}$ between session 1 and session 2 (RQ1a and RQ2) and over the course of four training sessions (RQ1b). Separate one-way MANOVA and ANOVA tests were done to analyze the differences in performance between group $\mathrm{L}$ and group $\mathrm{C}$ in their first training sessions (RQ3) and their second training sessions (RQ4) on each task. To minimize the bias of extreme outliers on the comparison of means, we excluded the performances with a $z$-score larger than 3.29. To reduce the probability of failing to identify a genuine effect (a type II error), a $P$ value $\leq 0.05$ was considered statistically significant, and a $P$ value between 0.05 and 0.07 indicates a considerable tendency of the results.

\section{Results}

Performance improvement within each group

Overall, the performances within both groups on the simulator tasks improved considerably after two and four training sessions compared to the baseline performance in the first training session (Figs. 3, 4). Between the first and second training sessions in each IBP technique, the MANOVA tests for group $\mathrm{C}$ showed a significant improvement in performance on the PA task and the EB L1 task. For group $\mathrm{L}$ the MANOVA tests showed significant improvement in performance on the PA task, VRC task, and EB L1 task between the first and second training sessions in both IBP techniques. Over four simulator training sessions the performance of group C on the EB L1 task improved significantly, while a considerable tendency was found for group $\mathrm{L}$ on their performance of the $\mathrm{CN}$ task over four sessions $(P=0.053)$.

The ANOVA tests showed that group $\mathrm{C}$ performed the $\mathrm{CN}$ task and the PA task in laparoscopy session 2 in 
Fig. 3 Means and standard deviations for the $\mathrm{CN}$ task
Fig. 4 Means and standard deviations for the VRC task
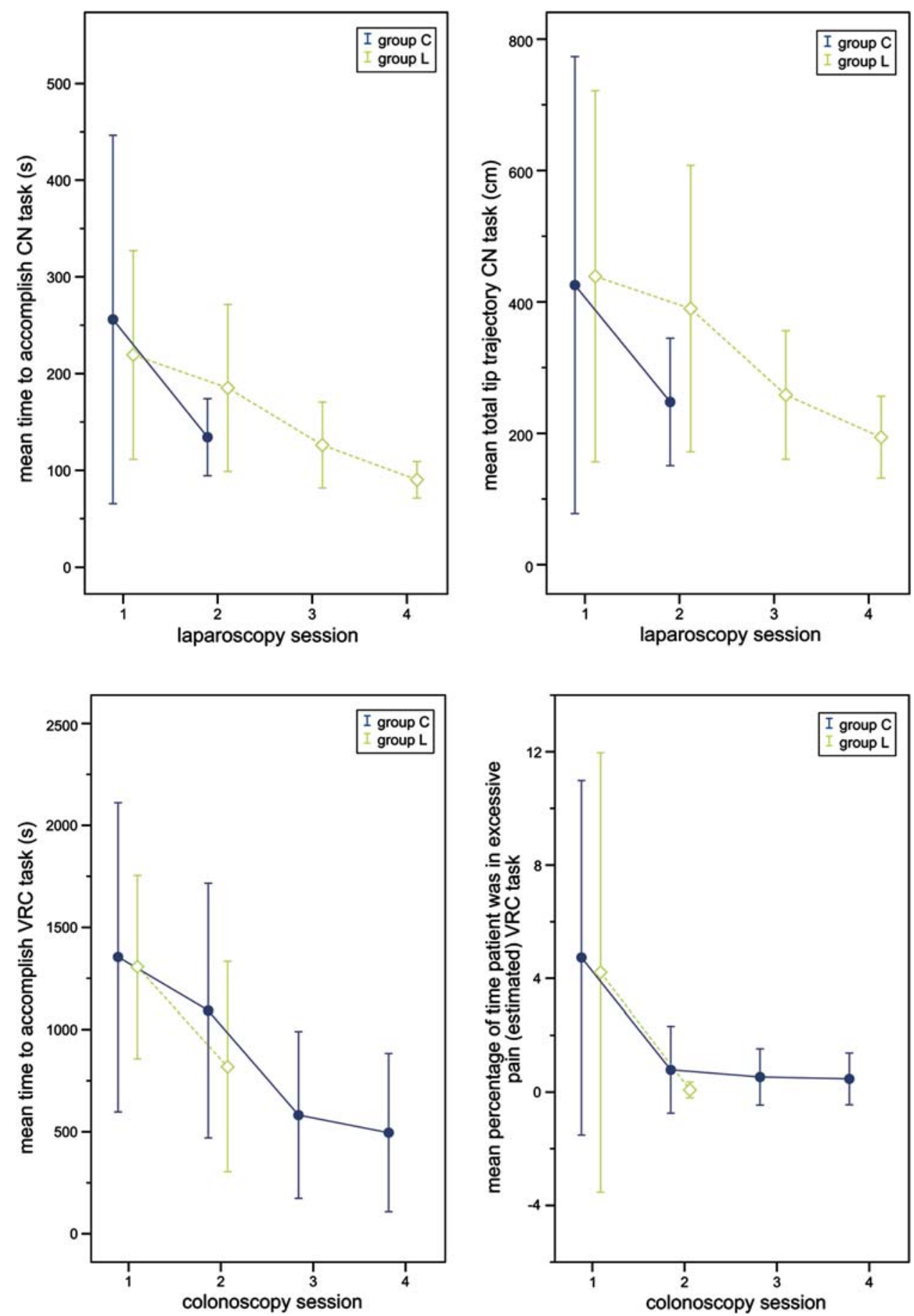

significantly less time than in laparoscopy session 1, and a considerable tendency was found for the total tip trajectory $(P=0.061)$ for the $\mathrm{CN}$ task. They performed the EB L1 task in both colonoscopy session 2 and over the course of four colonoscopy sessions in significantly less time and with fewer wall collisions than in colonoscopy session 1. In colonoscopy session 2 group C performed the VRC I-3 task with a lower percentage of time the virtual patient was in excessive pain than in session 1. Over four colonoscopy sessions, they required less time to perform the VRC I-3 task, and they did so with a lower percentage of time the virtual patient was in excessive pain compared to colonoscopy session 1. Group L performed the PA task in both laparoscopy session 2 and over four laparoscopy sessions in significantly less time, with a shorter total tip trajectory than in laparoscopy session 1 . In addition, a considerable tendency was found for the number of lost arrows ( $p=0.064)$ in laparoscopy session 2 compared to session 
1. On the $\mathrm{CN}$ task group $\mathrm{L}$ did not improve their performance between session 1 and session 2, but over the four laparoscopy sessions they did perform the $\mathrm{CN}$ task in significantly less time, with a shorter total tip trajectory and with fewer targets lost out of view. In colonoscopy session 2, group L performed the EB L1 task and the VRC I-3 task in significantly less time than in colonoscopy session 1.

Influence of colonoscopy training on performance of laparoscopy tasks

To assess whether colonoscopy training has an influence on the performance of basic laparoscopy tasks, the performances of group L were compared with the performances of group C for laparoscopy session 1 and laparoscopy session 2 (Fig. 3). The MANOVA tests showed a significant difference in performance in the second laparoscopy training session on the $\mathrm{CN}$ task between group $\mathrm{L}$ and group C. This holds for the total tip trajectory of the CN task, whereas a considerable tendency was found for the time to accomplish the $\mathrm{CN}$ task $(P=0.051)$; group $\mathrm{C}$ performed the $\mathrm{CN}$ task better than group L. No significant differences or considerable tendencies were found between the performances of the two groups for the PA task in laparoscopy session 2 .

Influence of laparoscopy training on performance of colonoscopy tasks

To assess whether laparoscopy training has an influence on the performance of basic colonoscopy tasks, the performances of group L were compared with the performances of group $\mathrm{C}$ for colonoscopy session 1 and colonoscopy session 2. No significant differences were found in the first and second colonoscopy sessions between the performances of group $\mathrm{L}$ and group $\mathrm{C}$ over all performance scores (MANOVA) or on the separate scores (ANOVA) (Fig. 4). Similarly, no significant differences or considerable tendencies were found between the performances on the EB L1 task or the VRC I-3 task by group C and group L in their first colonoscopy session. The same holds for the comparison between the performances of the two groups on the EB L1 task and the VRC I-3 task in colonoscopy session 2.

\section{Discussion}

Laparoscopy and colonoscopy are two commonly practiced image-based procedures, the basic skills of which can be well trained preclinically on VR simulators [9-12]. Current trends and novelties in technology and surgical techniques, such as NOTES, increase the need for transfer of knowledge and skills among specialists in both techniques [14, 18, 19]. However, knowledge of the interaction between the dedicated skills for these two image-based surgical techniques is limited. We therefore explored the influence of colonoscopy training on the performance of basic laparoscopy tasks (and vice versa) by comparing the laparoscopy performances of a group of naïve endoscopists who had prior training in colonoscopy with the performances of a group of naïve endoscopists without this experience (and vice versa).

First of all, we needed to verify whether the two groups improved over the course of the simulator training for both techniques (RQ1 and RQ2). By practice on the GI Mentor and SimSurgery SEP simulators, task-specific skills improved considerably over four training sessions. The range in performance scores also decreased over the course of the training sessions. These findings match with previously published similar studies: medical trainees with no laparoscopy or flexible endoscopy experience improved their task performance considerably over the course of a VR simulator-based training program [11-13, 20, 21]. In addition, this study shows that four in-between training sessions in another image-based technique do not impinge on this effect.

The third research question relates to the influence of one colonoscopy training session on the performance of basic laparoscopy tasks (and vice versa) (RQ3 in Table 1). To this end, the performances of group L in their first laparoscopy training session (after performing one colonoscopy training session) were compared with the performances of group $\mathrm{C}$ in their first laparoscopy training session (without any colonoscopy experience) (and vice versa) (Fig. 2). The results showed similar scores for group $\mathrm{C}$ and group $\mathrm{L}$ on both the laparoscopy tasks and the colonoscopy tasks; no notable differences in performance were found. By comparing the performances of group $\mathrm{C}$ and group L in their second training session, the influence of colonoscopy training on the performances of basic laparoscopy tasks (and vice versa) was assessed (RQ4 in Table 1). In the applied cross-over study design the only difference between group $\mathrm{L}$ and group $\mathrm{C}$ in their second laparoscopy training session was the amount of additional training on basic colonoscopy tasks (and vice versa) they had prior to their second laparoscopy training session (Fig. 2). The ANOVA test showed that in the second laparoscopy training session, the performances of group $\mathrm{C}$ on the $\mathrm{CN}$ task just surpassed the performances of group $\mathrm{L}$ (Fig. 3). No notable differences were found between the groups for the PA task in their second laparoscopy training session or for the EB L1 task and VRC I-3 task in their second colonoscopy training session (Fig. 4). These results imply that training in basic laparoscopy skills does not 
affect the performance of basic colonoscopy tasks. And training in basic colonoscopy skills appears to have, to a limited extent, a positive influence on the performance of a basic angled laparoscope navigation task. Skills are certainly not directly interchangeable between these two IBP types. Experience in basic colonoscopy tasks does not imply better performance of basic laparoscopy tasks, and experience in basic laparoscopy tasks does not mean superior performance of basic colonoscopy tasks.

This study was set up to explore the existing assumption that when learning a new IBP technique, it is advantageous to have experience in another IBP technique. Our findings do not corroborate this assumption. It is important to note, however, that the results also show that training in colonoscopy does not negatively affect performance of basic laparoscopy tasks (and vice versa). Several studies previously investigated the clinical performance of general surgeons and colorectal surgeons on flexible endoscopic intraluminal interventions, colonoscopy in particular, in comparison to gastroenterologists $[15,16]$. Most studies were retrospective and focused on clinical outcomes, such as intubation rate and complications. These studies confirmed that it is the amount of training and experience of the individual physician that predicts the safety, efficacy, and outcome of colonoscopy, and not the specialization of the physician or surgeon [15, 16]. To the best of our knowledge there are no studies that compared the clinical performance of gastroenterologists and general surgeons in laparoscopy. Nevertheless, in some countries gastroenterologists perform diagnostic laparoscopic procedures [17]. The study by Adamsen et al. (2005) presented a positive correlation between performances on a VR colonoscopy simulator and a VR laparoscopy simulator. Unfortunately, because of several major differences in the set-up of their study and the study presented here, a comparison of results is not possible. Adamsen et al. included 24 participants with different levels of expertise in either colonoscopy, laparoscopy, or both, but they did not distinguish between the background and expertise of the participants.

To fulfil the generally shared desire for objective proficiency assessment and to accomplish a shift toward more criterion-based training, a better understanding of IBPrelated skills and the interrelation of these skills is indispensable [22, 23]. Because of the growing number of procedures being performed image-based and the rise of IBP with increasing technical complexity, such as NOTES, there is great need to extend the knowledge on IBP skills and proficiency assessment [14, 18, 23]. Better understanding and more objective assessment of IBP skills is essential for the development of more effective training programs that can take the overall IBP proficiency level and individual training needs of the trainee into account.
The set-up of the present study had some limitations. Several participants remarked on their own accord that they experienced the VRC I-3 task and the $\mathrm{CN}$ task as being harder work than the EB L1 task and the PA task. The increased challenge on the VRC task and the CN task might have contributed to a stronger learning effect for these particular tasks [24], in comparison to the EB L1 task and the PA task. The study was rather complex and timeconsuming for the participating medical trainees; this impeded the inclusion of large numbers of participants within the available time frame. With a total sample size of 29 participants the post-hoc power for the between-group comparisons was 0.66 , which is close to the aimed power of 0.7 . In the analysis we applied a correction, aiming to minimize the probability of falsely failing to identify a genuine effect (type II error). Such a correction brings about an increase of the probability of a type I error (falsely identifying an effect); however, in view of the exploratory aim of this study, this is acceptable. The size of the effect could also be smaller than assumed for the analysis based on previous studies, requiring inclusion of a substantially larger group of participants to be detected. However, if the effect of the interaction of skills between different types of IBP would indeed be small to medium for naïve endoscopists, this would not affect our conclusions.

\section{Conclusions}

This study shows that training in basic colonoscopy tasks does not affect performance of basic laparoscopy tasks (and vice versa). A minor transfer of basic psychomotor skills was found from training in basic colonoscopy skills to the performance of basic laparoscopy tasks, but only for angled laparoscope navigation. Thus, skills required to perform basic laparoscopy and colonoscopy tasks are not directly interchangeable. Training and assessment of IBP type-specific skills should therefore focus on each type of task independently. The minor difference in performance was found for the $\mathrm{CN}$ task, which involves complex spatial navigation. The influence of separate psychometric abilities on the performance of image-based procedures and the transferability of skills between different types of IBP therefore needs to be studied further. Future research should also increase the knowledge on the transfer of skills for physicians who are experienced in one IBP type and would like to become proficient in another type of IBP.

Acknowledgments This work was supported in part by a grant (to S. N. B.) from the Scientific Fund of the Catharina Hospital Eindhoven. The authors are grateful to all participants for their engagement in this study, as well as to SimSurgery, Xitact/Mentice, and Simbionix for providing equipment support, and Cees Schot and Guy van Dael (Catharina Hospital Eindhoven) for technical and 
audiovisual support. In addition, they thank Sanne Botden and Jeroen Heemskerk (Catharina Hospital Eindhoven) for support in carrying out the simulator training, and Ruth Mugge (Delft University of Technology) for providing statistical advice. Finally, the authors thank the anonymous reviewers of the journal for their extensive and very valuable comments on the manuscript.

Open Access This article is distributed under the terms of the Creative Commons Attribution Noncommercial License which permits any noncommercial use, distribution, and reproduction in any medium, provided the original author(s) and source are credited.

\section{References}

1. Hanna G, Cuschieri A (2001) Image display technology and image processing. World J Surg 25:1419-1427

2. Satava RM (2001) Information age technologies for surgeons: overview. World J Surg 25:1408-1411

3. Buzink SN, Goossens RHM, Jakimowicz JJ et al. (2006) Imagebased surgical proficiency: reflection on human factors. In Pikaar R, Konigsveld E, Settels P (eds) Meeting diversity in ergonomics proceedings of the 16th world congress on Ergonomics of the International Ergonomics Association, Elsevier Science, Maastricht, The Netherlands

4. Berguer R (1999) Surgery and ergonomics. Arch Surg 134:10111016

5. Satava RM, Ellis SR (1994) Human interface technology-an essential tool for the modern surgeon. Surg Endosc 8:817-820

6. Reyes DA, Tang B, Cuschieri A (2006) Minimal access surgery (MAS)-related surgeon morbidity syndromes. Surg Endosc 20:113

7. Tassios P, Ladas S, Grammenos I et al (1999) Acquisition of competence in colonoscopy: the learning curve of trainees. Endoscopy 31:702-706

8. Gallagher A, Smith C (2003) From the operating room of the present to the operating room of the future. Human-factors lessons learned from the minimally invasive surgery revolution. Semin Laparosc Surg 10:127-139

9. Dunkin B, Adrales GL, Apelgren K et al (2007) Surgical simulation: a current review. Surg Endosc 21:357-366
10. Seymour NE (2008) VR to OR: a review of the evidence that virtual reality simulation improves operating room performance. World J Surg 32:182-188

11. Buzink SN, Koch AD, Heemskerk J et al (2007) Acquiring basic endoscopy skills by training on the GI Mentor II. Surg Endosc 21:1996-2003

12. Buzink SN, Goossens RHM, de Ridder H et al. (in press) Training of basic laparoscopy skills on SimSurgery SEP. Minim Invasive Ther Allied Technol

13. Buzink SN, Botden SMBI, Heemskerk J et al (2009) Camera navigation and tissue manipulation; are these laparoscopic skills related? Surg Endosc 23:750-757

14. Morales MP, Mancini GJ, Miedema BW et al (2008) Integrated flexible endoscopy training during surgical residency. Surg Endosc 22:2013-2017

15. Mehran A, Jaffe P, Efron J et al (2003) Colonoscopy: why are general surgeons being excluded? Surg Endosc 17:1971-1973

16. Wexner SD, Forde KA, Sellers G et al (1998) How well can surgeons perform colonoscopy? Surg Endosc 12:1410-1414

17. Adamsen S, Funch-Jensen PM, Drewes AM et al (2005) A comparative study of skills in virtual laparoscopy and endoscopy. Surg Endosc 19:229-234

18. Al-Akash M, Boyle E, Tanner WA (2009) Training on N.O.T.E.S.: from history we learn. Surg Oncol 18:111-119

19. Onders RP (2003) The utility of flexible endoscopy during advanced laparoscopy. Semin Laparosc Surg 10:43-48

20. Ferlitsch A, Glauninger P, Gupper A et al (2002) Evaluation of a virtual endoscopy simulator for training in gastrointestinal endoscopy. Endoscopy 34:698-702

21. Eversbusch A, Grantcharov TP (2004) Learning curves and impact of psychomotor training on performance in simulated colonoscopy: a randomized trial using a virtual reality endoscopy trainer. Surg Endosc 18:1514-1518

22. Roberts KE, Bell RL, Duffy AJ (2006) Evolution of surgical skills training. World J Gastroenterol 12:3219-3224

23. Jakimowicz JJ, Cuschieri A (2005) Time for evidence-based minimal access surgery training — simulate or sink. Surg Endosc 19:1-3

24. Ali MR, Mowery Y, Kaplan B et al (2002) Training the novice in laparoscopy-more challenge is better. Surg Endosc 16:17321736 